\title{
Evolution of proton single-particle states in neutron-rich Sb isotopes beyond $N=82$
}

A. Jungclaus, ${ }^{1,}{ }^{*}$ J. M. Keatings, ${ }^{2}$ G. S. Simpson, ${ }^{3}$ H. Naïdja, ${ }^{4}$ A. Gargano, ${ }^{5}$ S. Nishimura, ${ }^{6}$ P. Doornenbal, ${ }^{6}$ G. Gey, ${ }^{3,7,6}$ G. Lorusso, ${ }^{6}$ P.-A. Söderström, ${ }^{6}$ T. Sumikama, ${ }^{8}$ J. Taprogge ${ }^{1,9,6}$ Z. Y. Xu, ${ }^{6}$ H. Baba, ${ }^{6}$ F. Browne, ${ }^{10,6}$ N. Fukuda, ${ }^{6}$ N. Inabe, ${ }^{6}$ T. Isobe, ${ }^{6}$ H. S. Jung, ${ }^{11}$ D. Kameda, ${ }^{6}$ G. D. Kim, ${ }^{12}$ Y.-K. Kim, ${ }^{12,13}$ I. Kojouharov, ${ }^{14}$ T. Kubo, ${ }^{6}$ N. Kurz, ${ }^{14}$ Y. K. Kwon, ${ }^{12}$ Z. Li, ${ }^{15}$ H. Sakurai, ${ }^{6,16}$ H. Schaffner, ${ }^{14}$ Y. Shimizu, ${ }^{6}$ H. Suzuki, ${ }^{6}$ H. Takeda, ${ }^{6}$ Z. Vajta, ${ }^{17}$ H. Watanabe, ${ }^{6}$ J. Wu, ${ }^{15,6}$ A. Yagi, ${ }^{18}$ K. Yoshinaga, ${ }^{19}$ S. Bönig, ${ }^{20}$ J.-M. Daugas, ${ }^{21}$ R. Gernhäuser, ${ }^{22}$ S. Ilieva, ${ }^{20}$ T. Kröll, ${ }^{20}$ A. Montaner-Piza, ${ }^{23}$ K. Moschner, ${ }^{24}$ D. Mücher, ${ }^{22}$ H. Nishibata, ${ }^{18}$ A. Odahara, ${ }^{18}$ R. Orlandi, ${ }^{25}$ M. Scheck, ${ }^{26}$ K. Steiger, ${ }^{22}$ and A. Wendt ${ }^{24}$

${ }^{1}$ Instituto de Estructura de la Materia, CSIC, E-28006 Madrid, Spain

${ }^{2}$ School of Computing, Engineering, and Physical Sciences, University of the West of Scotland, Paisley PA1 2BE, United Kingdom

${ }^{3}$ LPSC, Université Joseph Fourier Grenoble 1, CNRS/IN2P3, Institut National Polytechnique de Grenoble, F-38026 Grenoble Cedex, France

${ }^{4}$ Laboratoire de Physique Mathematique et Subatomique, Constantine 1 University, Constantine 25000, Algeria

${ }^{5}$ Istituto Nazionale di Fisica Nucleare, Complesso Universitario di Monte S. Angelo, I-80126 Napoli, Italy

${ }^{6}$ RIKEN Nishina Center, RIKEN, 2-1 Hirosawa, Wako-shi, Saitama 351-0198, Japan

${ }^{7}$ Institut Laue-Langevin, B.P. 156, F-38042 Grenoble Cedex 9, France

${ }^{8}$ Department of Physics, Tohoku University, Aoba, Sendai, Miyagi 980-8578, Japan

${ }^{9}$ Departamento de Física Teórica, Universidad Autónoma de Madrid, E-28049 Madrid, Spain

${ }^{10}$ School of Computing, Engineering and Mathematics, University of Brighton, Brighton BN2 4GJ, United Kingdom

${ }^{11}$ Department of Physics, Chung-Ang University, Seoul 156-756, Republic of Korea

${ }^{12}$ Rare Isotope Science Project, Institute for Basic Science, Daejeon 305-811, Republic of Korea

${ }^{13}$ Department of Nuclear Engineering, Hanyang University, Seoul 133-791, Republic of Korea

${ }^{14}$ GSI Helmholtzzentrum für Schwerionenforschung GmbH, 64291 Darmstadt, Germany

${ }^{15}$ School of Physics and State Key Laboratory of Nuclear Physics and Technology, Peking University, Beijing 100871, China

${ }^{16}$ Department of Physics, University of Tokyo, Hongo 7-3-1, Bunkyo-ku, 113-0033 Tokyo, Japan

${ }^{17}$ Atomki, P.O. Box 51, Debrecen H-4001, Hungary

${ }^{18}$ Department of Physics, Osaka University, Machikaneyama-machi 1-1, Osaka 560-0043 Toyonaka, Japan

${ }^{19}$ Department of Physics, Faculty of Science and Technology, Tokyo University of Science, 2641 Yamazaki, Noda, Chiba, Japan

${ }^{20}$ Institut für Kernphysik, Technische Universität Darmstadt, D-64289 Darmstadt, Germany

${ }^{21}$ CEA, DAM, DIF, 91297 Arpajon cedex, France

${ }^{22}$ Physik Department E12, Technische Universität München, D-85748 Garching, Germany

${ }^{23}$ Instituto de Fsica Corpuscular, CSIC-Univ. of Valencia, E-46980 Paterna, Spain

${ }^{24}$ IKP, University of Cologne, D-50937 Cologne, Germany

${ }^{25}$ Advanced Science Research Center, Japan Atomic Energy Agency, Tokai, Ibaraki, 319-1195, Japan

${ }^{26}$ School of Engineering, University of the West of Scotland, Paisley PA1 2BE, United Kingdom

(Received 21 April 2020; revised 7 May 2020; accepted 28 August 2020; published 23 September 2020)

The $\beta$ decay of the semimagic Sn isotopes ${ }^{136,137,138} \mathrm{Sn}$ has been studied at the Radioactive Isotope Beam Factory at the RIKEN Nishina Center. The first experimental information on excited states was obtained for ${ }^{137} \mathrm{Sb}$ while, in the case of ${ }^{136} \mathrm{Sb}$, the established excitation scheme could be extended by ten previously unidentified levels. In the decay of the most-neutron-rich isotope ${ }^{138} \mathrm{Sn}$, two $\gamma$ rays were observed for the first time. The new experimental results, in combination with state-of-the-art shell-model calculations, provide the first information with respect to the evolution of the $0 g_{7 / 2}$ and $1 d_{5 / 2}$ proton single-particle states with increasing neutron number beyond $N=84$.

DOI: 10.1103/PhysRevC.102.034324

\section{INTRODUCTION}

The chain of $\mathrm{Sb}$ isotopes, with a single proton outside the closed $Z=50$ proton shell, has attracted for many years a special interest since it offers the unique possibility to study the

*andrea.jungclaus@csic.es evolution of the proton single-particle states in the $Z=50-82$ major shell over a wide range of neutron number. At present, excited-state information is available from the very-neutrondeficient isotope ${ }^{105} \mathrm{Sb}_{54}$, close to the presumably doubly magic ${ }^{100} \mathrm{Sn}$, up to the neutron-rich isotope ${ }^{135} \mathrm{Sb}_{84}$, which is situated beyond the $N=82$ shell closure. In particular, the experimentally observed variation of the excitation energies of the lowest-lying $7 / 2^{+}, 5 / 2^{+}$, and $11 / 2^{-}$states, which depend 


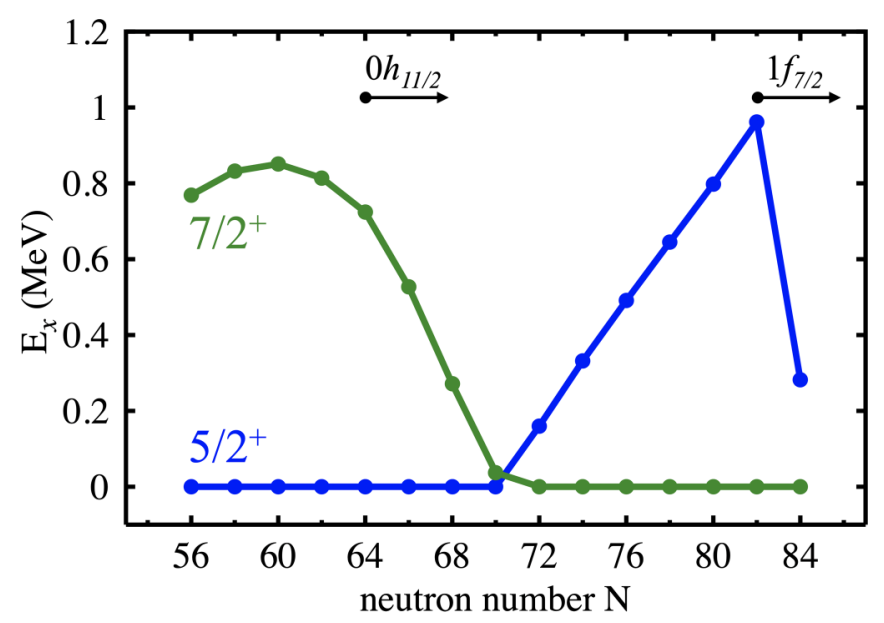

FIG. 1. Evolution of the experimental excitation energies of the first $5 / 2^{+}$and $7 / 2^{+}$states in the odd- $A$ Sb isotopes as a function of the neutron number $N$ (data taken from Ref. [6]).

on both the proton single-particle energies (SPEs) of the $0 g_{7 / 2}$, $1 d_{5 / 2}$, and $0 h_{11 / 2}$ orbitals and their coupling to the $2_{1}^{+}$and $3_{1}^{-}$states of the Sn cores, has been extensively discussed in the literature (e.g., see Refs. [1-5]). While all isotopes up to ${ }^{121} \mathrm{Sb}_{70}$ have a ground-state spin of $5 / 2^{+}$, as shown in Fig. 1, the $7 / 2^{+}$level becomes the ground state in all heavier $\mathrm{Sb}$ isotopes studied so far. The reason for this crossing is a smooth, nearly linear decrease of the binding energy of the $0 g_{7 / 2}$ proton orbital with respect to the $1 d_{5 / 2}$ orbital as soon as the $0 h_{11 / 2}$ neutron orbital is being filled above $N=64$, which is caused by a strongly attractive monopole interaction between the $0 g_{7 / 2}$ and $0 h_{11 / 2}$ orbitals. Above the $N=82$ neutron shell gap, when the neutrons start filling the $1 f_{7 / 2}$ orbital, the situation changes abruptly. The excitation energy of the $5 / 2^{+}$state suddenly drops from $962 \mathrm{keV}$ in ${ }^{133} \mathrm{Sb}_{82}$ to $282 \mathrm{keV}$ in ${ }^{135} \mathrm{Sb}_{84}[7,8]$, representing the inversion of the trend observed up to the shell closure. Qualitatively, this behavior can be understood considering the overlap of the radial wave functions of the protons and neutrons. Since the $\nu 0 h_{11 / 2}$ and $\pi 0 g_{7 / 2}$ orbitals are both nodeless, the overlap of their wave functions is larger as compared with that of the same neutron orbital with the $1 d_{5 / 2}$ proton orbital with one node. In contrast to the $v 0 h_{11 / 2}$ orbital below $N=82$, the $v 1 f_{7 / 2}$ being filled above the shell closure has one node and therefore a larger overlap with the $1 d_{5 / 2}$ proton orbital as compared with the $0 g_{7 / 2}$. Self-consistent calculations using the D1S Gogny force reproduce the crossing around $N=70$ as well as the decrease of the $5 / 2_{1}^{+}$state beyond $N=82[2,4]$. Also, shell-model calculations employing a realistic effective interaction are able to reproduce this drop of the $5 / 2_{1}^{+}$energy between ${ }^{133} \mathrm{Sb}$ and ${ }^{135} \mathrm{Sb}[9,10]$. They furthermore show that, due to the reduced neutron pairing above $N=82$, the $5 / 2_{1}^{+}$ state in ${ }^{135} \mathrm{Sb}$ is not a pure single-particle state but of admixed nature. This of course implies that the excitation energy of this state cannot be equated with the $\pi 1 d_{5 / 2}$ SPE.

Here, we report on the identification of excited states in the $N=85-87$ isotopes ${ }^{136-138} \mathrm{Sb}$, populated in the $\beta$ decay of ${ }^{136-138} \mathrm{Sn}$. The experimental results are compared with shell-model calculations using two different realistic effective interactions. Based on this comparison, the evolution of the proton SPE as a function of neutron number is traced.

\section{EXPERIMENT}

The experiment was performed at the RIBF, operated by the RIKEN Nishina Center for Accelerator-Based Science and the Center for Nuclear Study of the University of Tokyo, in the framework of the EURICA project [11,12]. Neutron-rich ${ }^{136-138} \mathrm{Sn}$ ions were produced in the fission of a ${ }^{238} \mathrm{U}$ beam with a kinetic energy of $345 \mathrm{MeV} / u$ and an average intensity of $8-10 \mathrm{pnA}$ on a Be target. The produced ions were identified in flight event by event by the BigRIPS separator [13] on the basis of measured energy loss $\Delta E$, time of flight (TOF), and magnetic rigidity $B \rho$ [14]. The BigRIPS setting was optimized for the transmission of ${ }^{135} \mathrm{Sn}$. In total about $6.2 \times 10^{5}{ }^{136} \mathrm{Sn}, 4.9 \times 10^{4}{ }^{137} \mathrm{Sn}$, and $2.4 \times 10^{3}{ }^{138} \mathrm{Sn}$ ions were identified, transported through the ZeroDegree spectrometer, and finally implanted into the wide-range active silicon strip stopper array for $\beta$ and ion detection (WAS3ABi) array positioned at the focal plane of the ZeroDegree spectrometer. The WAS3ABi detector $[11,12]$ consisted of eight closely packed double-sided silicon strip detectors (DSSSDs) with an area of $60 \times 40 \mathrm{~mm}^{2}$, a thickness of $1 \mathrm{~mm}$, and a segmentation of 40 horizontal and 60 vertical strips each. All decay events detected in WAS3ABi were stored and correlated off-line in space and time with the implanted ions. The $\gamma$ rays emitted following the $\beta$ decay of the radioactive nuclei were detected by the EURICA array, which comprised 84 germanium crystals (12 large-volume cluster detectors [15] from the former EUROBALL spectrometer [16]) arranged in a close geometry around the $\mathrm{Si}$ detectors. This geometry assured a high $\gamma$-ray detection efficiency of $8.5(4) \%$ [2.9(2)\%] for a $\gamma$-ray with an energy of $1 \mathrm{MeV}(4 \mathrm{MeV})$ after applying appropriate add-back algorithms (summing the energies of neighboring crystals). Note that experimental results for the $\beta$ decay of other nuclei produced in the same experiment have previously been presented in Refs. [17-20].

\section{DATA ANALYSIS AND RESULTS}

Figure 2 shows the $\gamma$-ray spectra observed in prompt coincidence with the first decay event after the implantation of ${ }^{136} \mathrm{Sn},{ }^{137} \mathrm{Sn}$, and ${ }^{138} \mathrm{Sn}$ ions, respectively, in WAS3ABi. The time window between the implantation and the decay was limited to $1.050,0.69$, and $0.42 \mathrm{~s}$, corresponding for each isotope to three times the half-life [21]. For ${ }^{136,137} \mathrm{Sn}$, only those events in which the decay occurred within the same $1 \mathrm{~mm}^{2}$ $\mathrm{Si}$ pixel in which the ion was implanted were considered, while for ${ }^{138} \mathrm{Sn}$, of which much fewer ions were implanted, a less strict spatial correlation was applied. In this case, all events in which the implantation and the decay were detected in the same Si detector with a distance of less than $1.5 \mathrm{~mm}$ between them were taken into account. The two most intense lines in the spectra shown in Fig. 2, at energies of $535 \mathrm{keV}$ in part (a) and $84 \mathrm{keV}$ in part (b), are truncated in that figure to allow for a better visibility of the many weaker transitions in the spectra. $\gamma$ rays emitted following the daughter decays 


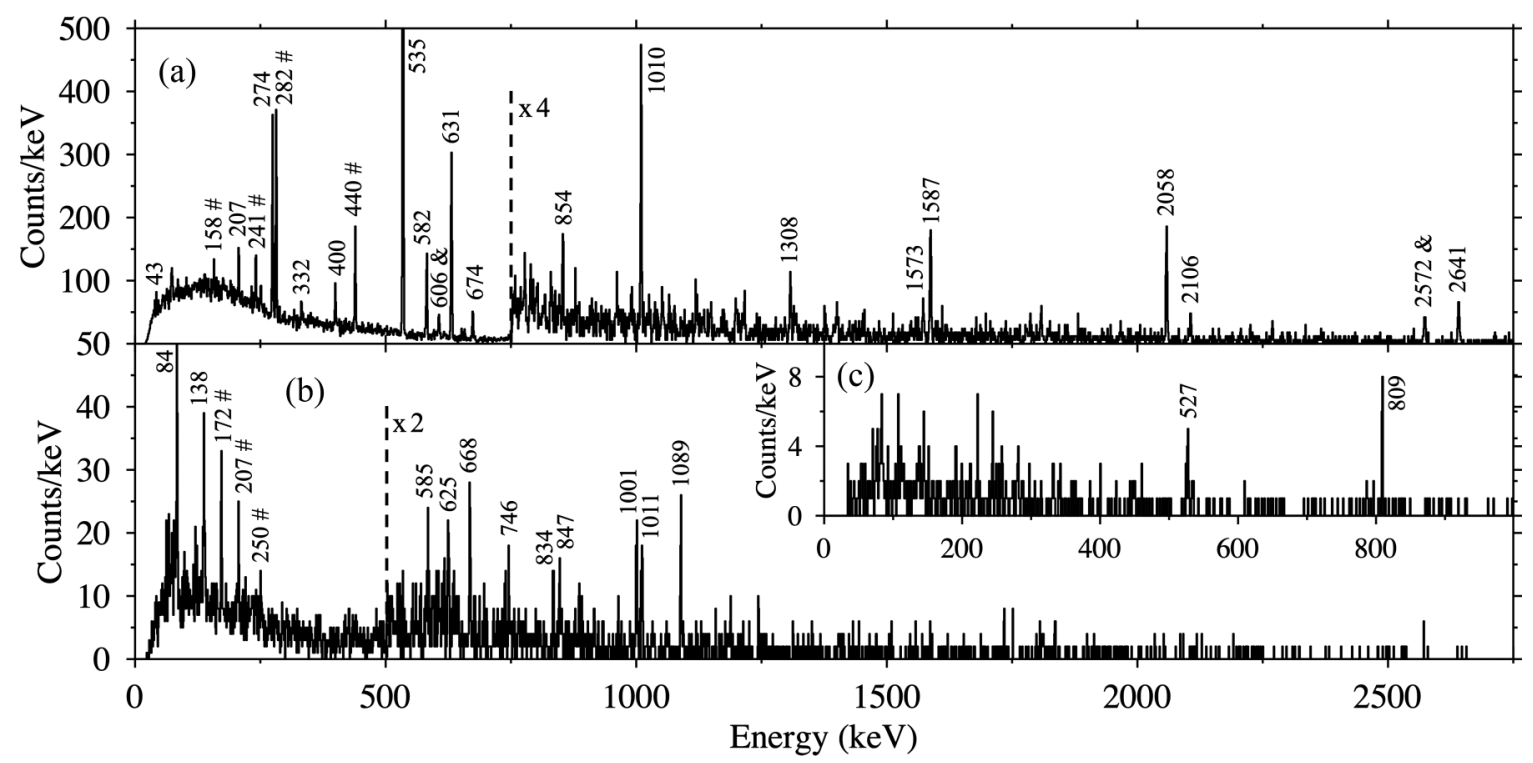

FIG. 2. $\gamma$-ray spectra observed in prompt coincidence with the first $\beta$ decay detected in WAS3ABi after the implantation of (a) ${ }^{136} \mathrm{Sn}$, (b) ${ }^{137} \mathrm{Sn}$, and (c) ${ }^{138} \mathrm{Sn}$ ions. The time interval between the implantation and the decay was limited to three times the half-life for each isotope. The most intense lines are labeled by their energies. $\gamma$ rays emitted following $\beta$-delayed neutron emission are labeled by “\#," while the symbol " $\&$ " marks the most intense $\gamma$ rays emitted following daughter decays.

have been identified by comparing spectra sorted for different time intervals after the implantation. Comparing spectra sorted with different requirements with respect to the spatial correlation, a total of 27,15 , and $2 \gamma$ rays were assigned to follow the decays of ${ }^{136} \mathrm{Sn},{ }^{137} \mathrm{Sn}$, and ${ }^{138} \mathrm{Sn}$, respectively, and their energies and relative intensities are listed in Table I. We note that many additional weaker lines are observed following the decays of ${ }^{136,137} \mathrm{Sn}$ when less strict conditions on the spatial correlation between implantation and decay are applied. However, since no coincidence relations could be established for these less-intense transitions, it was not possible to place them in the level schemes of ${ }^{136,137} \mathrm{Sb}$. The decay curves of all three isotopes were fit with Bateman equations including the second-generation (daughter) decays. From these fits, half-lives of $T_{1 / 2}=361(5) \mathrm{ms}, T_{1 / 2}=238(8) \mathrm{ms}$, and $T_{1 / 2}=142(12) \mathrm{ms}$ were determined for the decays of ${ }^{136} \mathrm{Sn}$, ${ }^{137} \mathrm{Sn}$, and ${ }^{138} \mathrm{Sn}$, respectively. These values are in agreement with the results reported in Ref. [21] based on the same data set. From the fits of the decay curves, also the number of $\beta$ decays is obtained for each isotope, which allows us to convert the observed $\gamma$-ray intensities into absolute intensities per decay. The conversion factors are quoted in the caption of Table I. In the following sections, the construction of the level schemes of the $\beta$-decay daughters ${ }^{136,137,138} \mathrm{Sb}$ will be discussed separately for each of the three cases.

\section{A. The ${ }^{136} \mathrm{Sn} \rightarrow{ }^{136} \mathrm{Sb}$ decay}

So far, the only experimental information about excited states in ${ }^{136} \mathrm{Sb}$ comes from isomer spectroscopy [22-24]. First, the observation of a $173 \mathrm{keV} \gamma$ ray following the production of ${ }^{136} \mathrm{Sb}$ in the projectile fission of ${ }^{238} \mathrm{U}$ at GSI was reported and a half-life of $T_{1 / 2}=565(50) \mathrm{ns}$ for the isomeric state was measured [22]. Later, using $\gamma$-ray and conversion-electron spectroscopy at the Institut LaueLangevin, additional low-energy transitions were observed [23]. In that work, an isomeric state with spin $\left(6^{-}\right)$and a half-life of $T_{1 / 2}=480(100) \mathrm{ns}$ was proposed to decay via a $51.4-173.0-53.4 \mathrm{keV}$ cascade to the $\left(1^{-}\right)$ground state of ${ }^{136} \mathrm{Sb}$. Finally, based on a recent experiment performed at the RIBF facility at RIKEN, a slightly different cascade, namely 53-173-43 keV, was proposed to depopulate an isomeric $\left(6^{-}\right)$ state via excited $\left(4^{-}\right)$and $\left(2^{-}\right)$states $\left[T_{1 / 2}=489(40) \mathrm{ns}\right][24]$. In the $\gamma$-ray spectrum observed following the $\beta$ decay of ${ }^{136} \mathrm{Sn}$ shown in Fig. 2(a), a line at $173 \mathrm{keV}$ is clearly absent, while a small peak at $43 \mathrm{keV}$ is visible. In the study of the $\beta$-delayed neutron emission of ${ }^{136} \mathrm{Sn}$ in Ref. [25], four $\gamma$ rays with energies of 158, 241, 282, and $440 \mathrm{keV}$ were observed. All four lines are clearly visible in Fig. 2(a). Most of the remaining lines in this spectrum could be arranged in a level scheme of ${ }^{136} \mathrm{Sb}$ based on (i) $\gamma \gamma$-coincidence relations, (ii) $\gamma$-ray intensities, and (iii) $\gamma$-ray energy sums. Some examples of coincidence spectra are shown in Fig. 3. The transitions with highest intensities in ${ }^{136} \mathrm{Sb}$ are those with energies of 535 , 631 , and $274 \mathrm{keV}$. While the $631 \mathrm{keV} \gamma$ ray is clearly observed in coincidence with the $43 \mathrm{keV}$ transition known from isomer spectroscopy [24], see Fig. 3(a), this low-energy transition is not observed in coincidence with the much stronger $535 \mathrm{keV}$ $\gamma$ ray. The latter is therefore assigned as a ground-state transition, thus establishing a new state at an excitation energy of $535 \mathrm{keV}$. The energy sum of the 274 and $400 \mathrm{keV}$ transitions, which are observed in mutual coincidence [see Fig. 3(b)], equals that of the 631 and $43 \mathrm{keV}$ lines and furthermore also a $\gamma$ ray with the sum energy of $674 \mathrm{keV}$ is observed. Based on this information, new states at 274 and $674 \mathrm{keV}$ could be established. In the coincidence spectrum of the $274 \mathrm{keV}$ transition, in addition to the $400 \mathrm{keV} \gamma$ ray, two more lines at 778 and $794 \mathrm{keV}$ are visible. These are assigned to the decays 
TABLE I. Energies and relative intensities, normalized for each isotope to the most intense transition, of $\gamma$ rays observed following the $\beta$ decays of ${ }^{136,137,138} \mathrm{Sn}$. The errors quoted for the $\gamma$-ray energies reflect the uncertainties from the fit. The systematic uncertainties from the calibration can be estimated to $0.2 \mathrm{keV}$ for energies below $1 \mathrm{MeV}$ and $0.6 \mathrm{keV}$ for energies above. To obtain the absolute intensity per 100 decays, the relative intensities have to be multiplied by the following factors: 0.356 for ${ }^{136} \mathrm{Sn}, 0.144$ for ${ }^{137} \mathrm{Sn}$, and 0.21 for ${ }^{138} \mathrm{Sn}$.

\begin{tabular}{|c|c|c|c|}
\hline$E_{\gamma}(\mathrm{keV})$ & $I_{\gamma}^{\mathrm{rel}}$ & $E_{i}(\mathrm{keV})$ & $E_{f}(\mathrm{keV})$ \\
\hline \multicolumn{4}{|c|}{${ }^{136}$ Sn decay: } \\
\hline $42.5(5)$ & & 43 & 0 \\
\hline $157.6(2)^{\mathrm{a}}$ & $2.7(5)$ & & \\
\hline $207.0(1)$ & $6.3(6)$ & 250 & 43 \\
\hline $241.0(1)^{\mathrm{a}}$ & $8.6(8)$ & & \\
\hline $250.1(1)$ & $5.4(8)$ & 250 & 0 \\
\hline 274.4(1) & $23(2)$ & 274 & 0 \\
\hline $281.7(1)^{\mathrm{a}}$ & $30(2)$ & & \\
\hline $332.2(1)$ & $3.7(5)$ & 582 & 250 \\
\hline $399.6(1)$ & $8.0(7)$ & 674 & 274 \\
\hline $439.5(1)^{\mathrm{a}}$ & $14(1)$ & & \\
\hline $534.7(1)$ & $100(5)$ & 535 & 0 \\
\hline $535(1)$ & $0.8(6)$ & 1068 & 535 \\
\hline $582.1(1)$ & $15(1)$ & 582 & 0 \\
\hline 631.4(1) & $34(2)$ & 674 & 43 \\
\hline 674.1(1) & $5.6(7)$ & 674 & 0 \\
\hline $778.0(2)$ & $3.0(5)$ & 1052 & 274 \\
\hline $793.9(3)$ & $2.4(5)$ & 1068 & 274 \\
\hline $853.8(2)$ & $3.0(6)$ & 854 & 0 \\
\hline 1009.7(1) & $10.6(8)$ & 1052 & 43 \\
\hline $1307.6(2)$ & $3.8(6)$ & 1890 & 582 \\
\hline $1572.5(3)$ & $3.0(6)$ & 2641 & 1068 \\
\hline 1587.4(1) & $6.9(9)$ & 2641 & 1052 \\
\hline $1787.0(3)$ & $1.7(5)$ & 2641 & 854 \\
\hline 2058.3(1) & $9.4(12)$ & 2641 & 582 \\
\hline 2106.2(3) & $1.5(4)$ & 2641 & 535 \\
\hline $2366.5(3)$ & $0.5(2)$ & 2641 & 274 \\
\hline $2640.9(2)$ & $4.3(7)$ & 2641 & 0 \\
\hline \multicolumn{4}{|c|}{${ }^{137}$ Sn decay: } \\
\hline $83.7(1)$ & 100(9) & 84 & 0 \\
\hline 137.7(1) & $47(7)$ & 221 & 84 \\
\hline $172.3(1)^{\mathrm{a}}$ & $40(5)$ & & \\
\hline $205.9(4)^{\mathrm{a}}$ & $27(5)$ & & \\
\hline $250.2(4)^{\mathrm{a}}$ & $8(4)$ & & \\
\hline $584.5(3)$ & $25(5)$ & 668 & 84 \\
\hline $625.4(3)$ & $21(5)$ & 847 & 221 \\
\hline $668.2(2)$ & $33(5)$ & 668 & 0 \\
\hline $745.8(3)$ & $12(4)$ & 1835 & 1089 \\
\hline $834.2(3)$ & $16(5)$ & 1835 & 1001 \\
\hline $847.3(2)$ & $28(5)$ & 847 & 0 \\
\hline $1001.2(2)$ & $35(6)$ & 1001 & 0 \\
\hline $1011.3(2)$ & $37(7)$ & 1095 & 84 \\
\hline $1089.0(2)$ & $31(5)$ & 1089 & 0 \\
\hline $1835.7(5)$ & $14(4)$ & 1835 & 0 \\
\hline \multicolumn{4}{|c|}{${ }^{138}$ Sn decay: } \\
\hline \multicolumn{4}{|c|}{$527(1)$} \\
\hline $809(1)$ & $100(23)$ & $809+x$ & $x$ \\
\hline
\end{tabular}

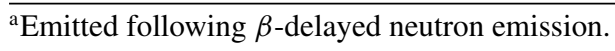

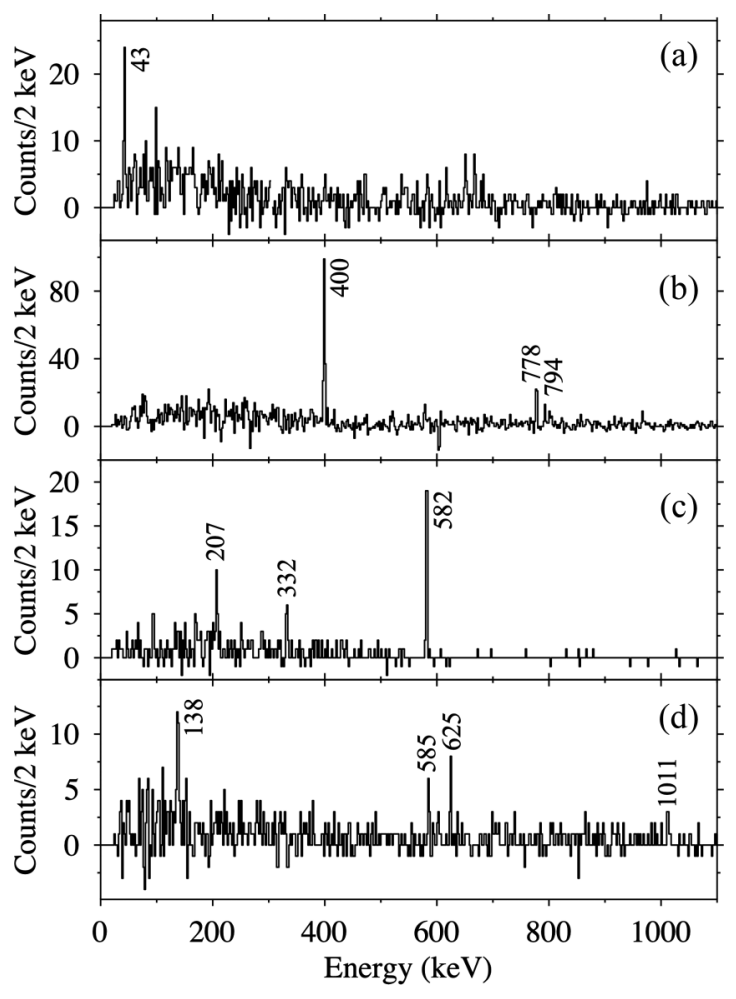

FIG. 3. Spectra of $\gamma$ rays observed in coincidence with the (a) $631 \mathrm{keV}$, (b) $274 \mathrm{keV}$, and (c) $2058 \mathrm{keV}$ transitions in ${ }^{136} \mathrm{Sb}$ and the (d) $84 \mathrm{keV}$ transition in ${ }^{137} \mathrm{Sb}$. All $\gamma$ rays emitted promptly after the first or second decay detected in WAS3ABi within a time window of $3 \times T_{1 / 2}$ after the implantation of a ${ }^{136} \mathrm{Sn}$ or ${ }^{137} \mathrm{Sn}$ ion, respectively, were considered.

of two new states at excitation energies of 1052 and 1068 $\mathrm{keV}$, the former decaying in parallel via a $1010 \mathrm{keV}$ transition to the $43 \mathrm{keV}$ level. Finally, several $\gamma$ rays with energies above $1.5 \mathrm{MeV}$ were observed following the decay of ${ }^{136} \mathrm{Sn}$. Seven of these transitions could be assigned to the decay of one single, strongly populated excited state at an energy of $2641 \mathrm{keV}$, only $250 \mathrm{keV}$ below the neutron separation energy of $S_{n}=2888(6) \mathrm{keV}$ [26]. Besides the ground state, the excited states at $274,535,582,854,1052$, and $1068 \mathrm{keV}$ are populated in its decay. To illustrate the decay of this highly excited state, the spectrum observed in coincidence with the $2058 \mathrm{keV} \gamma$ ray is shown in Fig. 3(c). Besides the $582 \mathrm{keV}$ ground-state transition, $\gamma$ rays with 207 and $332 \mathrm{keV}$ are observed, which connect the $582 \mathrm{keV}$ level to the first-excited state at $43 \mathrm{keV}$. The excitation scheme of ${ }^{136} \mathrm{Sb}$ established in the present work, which considerably extends the information available from isomeric decays and confirms the existence of the $43 \mathrm{keV}$ state proposed in Ref. [24], is shown in Fig. 4. Based on this scheme, observed $\beta$-feeding intensities were determined for all excited states from the $\gamma$-ray intensities listed in Table I. Note that, for all three studied isotopes, $\log f t$ values were calculated from the observed beta feeding by using the $Q_{\beta}$ values from Ref. [26] and half-lives of $T_{1 / 2}=$ $361(5) \mathrm{ms}, T_{1 / 2}=238(8) \mathrm{ms}$, and $T_{1 / 2}=142(12) \mathrm{ms}$ for the decays of ${ }^{136} \mathrm{Sn},{ }^{137} \mathrm{Sn}$, and ${ }^{138} \mathrm{Sn}$, respectively, which were determined in the present work, as discussed above. We note 


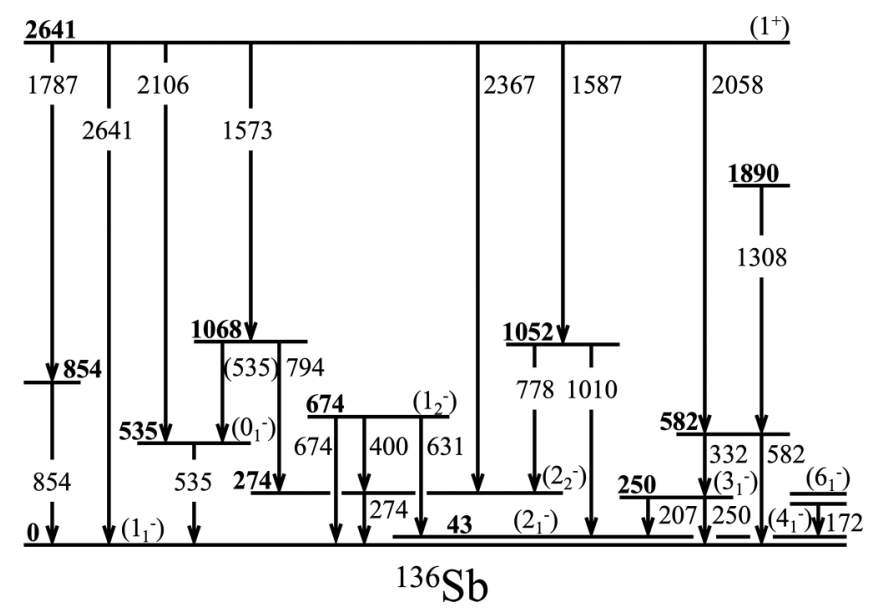

FIG. 4. Extended level scheme of ${ }^{136} \mathrm{Sb}$ established in the present work on the basis of coincidence relations between the $\gamma$ rays emitted following the $\beta$ decay of ${ }^{136} \mathrm{Sn}$. The $\left(4_{1}^{-}\right)$and $\left(6_{1}^{-}\right)$states, proposed in Ref. [24], but not populated in the $\beta$ decay of ${ }^{136} \mathrm{Sn}$, are included for completeness.

that, in some cases, in particular for low-lying excited states, the deduced $\log f t$ values, which are reported in Table II, may be affected by the Pandemonium effect [27] and therefore actually correspond to lower limits rather than values.

Experimental information, although rather limited, with respect to the spins of the newly established excited states, is provided by the $\log f t$ values. The $\beta$ decay of $Z=50$, $51, N>82$ isotopes is dominated by first-forbidden (ff) transitions, in particular the $v 1 f_{7 / 2} \rightarrow \pi 0 g_{7 / 2}$ and $\nu 1 f_{7 / 2} \rightarrow$ $\pi 0 d_{5 / 2}$ decays, in which low-lying $0^{-}, 1^{-}$states in ${ }^{136} \mathrm{Sb}$ are populated from the $0^{+}$ground state of ${ }^{136} \mathrm{Sn}$. This is in sharp contrast with the decay of the $N>82$ nuclei below the $Z=50$ gap in which the $\nu 0 g_{7 / 2} \rightarrow \pi 0 g_{9 / 2}$ GT decay prevails. For $Z=50,51$, the $\pi 0 g_{9 / 2}$ orbital is fully blocked and the $\nu 0 h_{9 / 2} \rightarrow \pi 0 h_{11 / 2}$ transition is therefore the only relevant allowed decay. In this decay, which is hindered by the small occupancy of the $v 0 h_{9 / 2}$ orbital in the ground state of ${ }^{136} \mathrm{Sn}$, high-lying $1^{+}$states in the daughter nucleus ${ }^{136} \mathrm{Sb}$ are populated.

In the decay of the even-even neighbor isotope ${ }^{134} \mathrm{Sn}$ to ${ }^{134} \mathrm{Sb}$, the $0^{-}$ground state and the $1_{2}^{-}$state at $885 \mathrm{keV}$ accumulate together $85 \%$ of the observed feeding intensity, and $\log f t$ values of 5.2 and 5.7, respectively, were determined in Ref. [8]. In the present experiment, the low energy of the $43 \mathrm{keV}$ ground-state transition prevents the determination of the feeding to the $\left(1_{1}^{-}\right)$and $\left(2_{1}^{-}\right)$states, since due to detector threshold effects no reliable efficiency calibration was available at this energy. For the strongly populated states at 535 and $674 \mathrm{keV}, \log f t$ values of 5.3(1) and 5.5(1) were obtained. In these cases, no additional lines were observed in the coincidence spectra with gates on the intense 535, 274, and $631 \mathrm{keV}$ transitions so that significant discrete feeding to these states can be excluded. We propose spin assignments of $0^{-}$and $1^{-}$for the 535 and $674 \mathrm{keV}$ states based on the observed decay to the $\left(1_{1}^{-}\right)$ground state of the first and the strongest decay branch to the $\left(2_{1}^{-}\right)$level of the second. For several other excited states, $\log f t$ values between 6.3 and 6.5
TABLE II. Excited states in ${ }^{136-138} \mathrm{Sb}$ populated in the $\beta$ decays of ${ }^{136-138} \mathrm{Sn}$ with the observed $\beta$-feeding intensities and the resulting $\log f t$ values [calculated using $Q_{\beta}$ values of 8.61(30), 10.27(40), and $9.36(118) \mathrm{MeV}$ [26] and half-lives of 361(5), 238(8), and 142 (12) $\mathrm{ms}$ for the decays of ${ }^{136} \mathrm{Sn},{ }^{137} \mathrm{Sn}$, and ${ }^{138} \mathrm{Sn}$, respectively] for each decay.

\begin{tabular}{|c|c|c|}
\hline$E_{x}(\mathrm{keV})$ & $I_{\beta}(\%)$ & $\log f t$ \\
\hline \multicolumn{3}{|l|}{${ }^{136} \mathrm{Sb}:$} \\
\hline \multicolumn{3}{|l|}{0} \\
\hline \multicolumn{3}{|l|}{$43(1)$} \\
\hline $250(1)$ & $2.9(4)$ & $6.4(1)$ \\
\hline $274(1)$ & $3.3(6)$ & $6.3(1)$ \\
\hline $535(1)$ & $34.8(18)$ & $5.3(1)$ \\
\hline $582(1)$ & $2.1(6)$ & $6.5(2)$ \\
\hline $674(2)$ & $16.8(8)$ & $5.5(1)$ \\
\hline $854(2)$ & $0.5(3)$ & $7.0(3)$ \\
\hline $1052(2)$ & $2.4(5)$ & $6.3(1)$ \\
\hline $1068(2)$ & $0.1(4)$ & \\
\hline $1890(2)$ & $1.4(2)$ & $6.3(1)$ \\
\hline $2641(2)$ & $9.7(7)$ & $5.2(1)$ \\
\hline \multicolumn{3}{|l|}{${ }^{137} \mathrm{Sb}:$} \\
\hline 0 & $19(16)^{\mathrm{a}}$ & $5.8(4)$ \\
\hline $84(1)$ & $11.8(37)^{\mathrm{a}}$ & $6.0(2)$ \\
\hline 221(1) & $5.5(15)^{\mathrm{a}}$ & $6.3(2)$ \\
\hline $668(1)$ & $8.3(11)$ & $6.0(1)$ \\
\hline $847(1)$ & $7.0(11)$ & $6.1(1)$ \\
\hline 1001(1) & $2.7(11)$ & $6.5(2)$ \\
\hline 1089(1) & $2.7(6)$ & $6.4(1)$ \\
\hline $1095(1)$ & $5.3(10)$ & $6.2(1)$ \\
\hline $1835(2)$ & $6.1(11)$ & $5.9(1)$ \\
\hline \multicolumn{3}{|l|}{${ }^{138}$ Sb: } \\
\hline $809+x$ & $21(5)$ & $5.2(4)$ \\
\hline
\end{tabular}

${ }^{\text {a Calculated assuming } M 1 \text { multipolarity for the } 84 \text { and } 138 \mathrm{keV}}$ transitions.

were obtained, indicating their population via ff transitions and suggesting spin values of $0^{-}, 1^{-}$. A special case is the state at $2641 \mathrm{keV}$, which is populated with a $\log f t$ value of 5.2(2). This level should not be significantly affected by the Pandemonium effect since it is only $250 \mathrm{keV}$ below the neutron separation energy. This finding is in strong contrast with the $\beta$ decay of ${ }^{134} \mathrm{Sn}$ in which three $1^{-}$levels in ${ }^{134} \mathrm{Sb}$ in the energy range 1.90-2.43 MeV were observed with $\log f t$ values between 7.0 and 7.2 [8]. The low $\log f t$ value may suggest that the $2641 \mathrm{keV}$ state is populated via a GT decay which would limit the possible spin values to $0^{+}$and $1^{+}$. Such an assignment would suggest spins of $0^{-}, 1^{-}$, or $2^{-}$ for all those excited states which are directly populated in the decay of the $2641 \mathrm{keV}$ state. Unfortunately, any further spin assignment will have to be model-based. We therefore refer to the discussion of the experimental results in comparison with shell-model calculations in Sec. IV.

\section{B. The ${ }^{137} \mathrm{Sn} \rightarrow{ }^{137} \mathrm{Sb}$ decay}

In the nucleus ${ }^{137} \mathrm{Sb}$, no excited states were known prior to the present work. In the $\gamma$-ray spectrum shown in Fig. 2(b), three lines, namely those at energies of 172, 207, and 


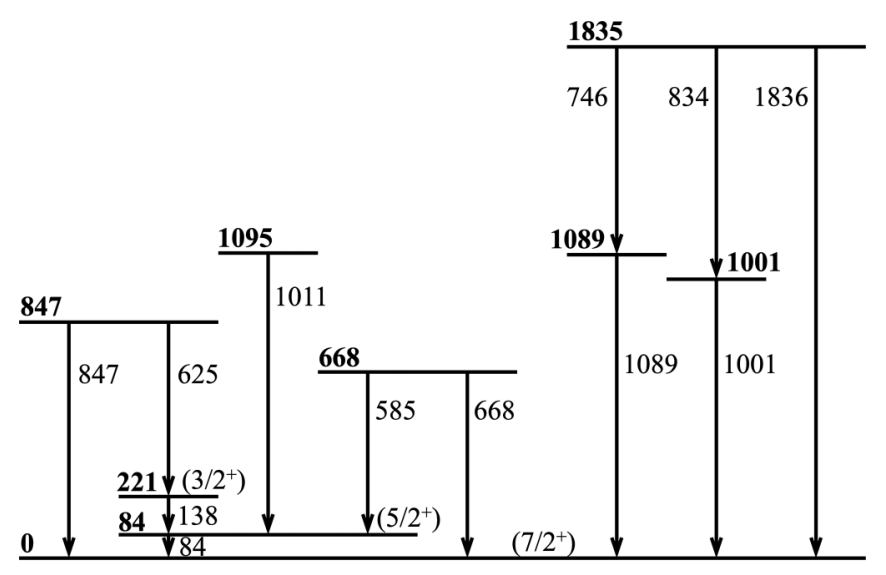

${ }^{137} \mathrm{Sb}$

FIG. 5. Proposed level scheme of ${ }^{137} \mathrm{Sb}$ established in the present work on the basis of $\gamma \gamma$ coincidence relations and energy sums.

$250 \mathrm{keV}$, correspond to the decays of the 215 and $250 \mathrm{keV}$ states in ${ }^{136} \mathrm{Sb}$, populated via $\beta$-delayed neutron emission (compare Fig. 4). The most intense lines in this spectrum are those at 84 and $138 \mathrm{keV}$. As demonstrated in Fig. 3(d), these two transitions are detected in prompt coincidence. Their order in the level scheme is fixed by the observation of additional $\gamma$ rays with energies of 585, 625, and $1011 \mathrm{keV}$ in coincidence with the $84 \mathrm{keV}$ transition, from which only the second is also coincident with the $138 \mathrm{keV} \gamma$ ray. This coincidence information allows us to establish five excited states at energies of 84, 221, 668, 847, and $1095 \mathrm{keV}$. Furthermore, the equality of the energy sums of the transitions with 746 and $1089 \mathrm{keV}$ on one side and 834 and $1001 \mathrm{keV}$ on the other suggests the existence of an additional level at an excitation energy of $1835 \mathrm{keV}$. Relaxing the strict correlation conditions applied for the spectra shown in Fig. 2, an $1836 \mathrm{keV} \gamma$ ray is also clearly observed following the decay of ${ }^{137} \mathrm{Sn}$, which most probably constitutes a parallel decay branch of the $1835 \mathrm{keV}$ state to the ground state. The excitation scheme of ${ }^{137} \mathrm{Sb}$, established for the first time in the present work, is shown in Fig. 5. In the $\beta$ decay of the lighter odd $\mathrm{Sn}$ isotopes ${ }^{133} \mathrm{Sn}$ and ${ }^{135} \mathrm{Sn}$, the $v 1 f_{7 / 2} \rightarrow \pi 0 g_{7 / 2}$ spin-flip, $\Delta J=0 \mathrm{ff}$ transition to the $7 / 2^{+}$ground state of the Sb daughter is the strongest decay branch with $\log f t$ values of 5.44 and 5.63, respectively [7,8]. In the decay of ${ }^{137} \mathrm{Sn}$, the absolute $\beta$ feeding to excited states sums to 49.4(47)\%; see Table II. Considering the $P_{n}$ values of $58(15) \%$ and 50(8)\%, which have been reported for this decay in Refs. [28,29], there would be very little room for any significant ground-state feeding. Therefore, in order to check these literature values, we estimated the $\beta$-delayed neutron emission probability by measuring the intensity of the $1726 \mathrm{keV}$ transition in ${ }^{137} \mathrm{Te}$ [30] following the implantation of ${ }^{137} \mathrm{Sn}$ and ${ }^{137} \mathrm{Sb}$, respectively. Here, all successive decays within $5 \mathrm{~s}$ after the implantation were considered and from the number of counts in the $1726 \mathrm{keV}$ line and the number of ${ }^{137} \mathrm{Sn}$ and ${ }^{137} \mathrm{Sb}$ decays, determined from fits of the corresponding decay curves, a value of $P_{n}=32(15) \%$ was obtained. Unfortunately, the uncertainties of both this estimate and the literature values are large so that the amount of ground-state feeding in the decay of ${ }^{137} \mathrm{Sn}$ remains an open question which only can be reliably answered when a more precise value of the $\beta$-delayed neutron emission probability becomes available in the future. Despite the lack of this important piece of information, we suggest a $7 / 2^{+}$ground-state spin also in ${ }^{137} \mathrm{Sb}$, in analogy to ${ }^{133} \mathrm{Sb}$ and ${ }^{135} \mathrm{Sb}$. This assignment is supported by the relative intensities of the $6^{+} \rightarrow 4^{+}, 4^{+} \rightarrow 2^{+}$, and $2^{+} \rightarrow 0^{+}$transitions in ${ }^{136} \mathrm{Te}$, emitted following $\beta$-delayed neutron emission and which are very similar to those observed in ${ }^{134} \mathrm{Te}$ following the decay of the $\left(7 / 2^{+}\right)$ground state of ${ }^{135} \mathrm{Sb}[30,31]$. The multipolarity of the $84 \mathrm{keV}$ transition is proposed to be $M 1\left(\alpha_{M 1}=1.03\right)$. The assumption of $E 2$ multipolarity $\left(\alpha_{E 2}=3.10\right)$ would imply a strong $(>40 \%)$ direct population of a $\left(3 / 2^{+}\right)$state at $84 \mathrm{keV}$ via a $\Delta J=2$ decay from the $7 / 2^{-}$ground state of ${ }^{137} \mathrm{Sn}$, while at the same time the direct feeding to the $\left(7 / 2^{+}\right)$ground state would be negligible. We therefore tentatively assign a spin of $5 / 2^{+}$to the $84 \mathrm{keV}$ state. Since the $\log f t$ values for all excited states, which have been established in the present work, are in the range expected for ff decays, $5 / 2^{+}, 7 / 2^{+}$, and $9 / 2^{+}$seem to be the most probable spin values for all of them. However, as noted before, all $\log f t$ values have to be considered as lower limits since various additional $\gamma$-ray transitions are observed in the singles spectra with less strict conditions but could not be placed in the level scheme due to the lack of coincidence information. The special case of the state at $221 \mathrm{keV}$ will be discussed in the next section.

\section{The ${ }^{138} \mathrm{Sn} \rightarrow{ }^{138}$ Sb decay}

The $\gamma$-ray spectrum observed following the decay of ${ }^{138} \mathrm{Sn}$ is shown in Fig. 2(c). Two lines are visible in this spectrum, a more intense one at $809 \mathrm{keV}$ and a weaker one at $527 \mathrm{keV}$. The absolute intensity of the $809 \mathrm{keV}$ transition is $21(5) \%$. In the $\beta n$ decay of ${ }^{136} \mathrm{Sn}$, the population of the $\left(5 / 2^{+}\right),\left(3 / 2^{+}\right)$, and $\left(1 / 2^{+}\right)$states of ${ }^{135} \mathrm{Sb}$ was reported [25]. It can therefore be expected that similar states are populated in the $\beta n$ decay of ${ }^{138} \mathrm{Sn}$. While there are hints of 84 and $137 \mathrm{keV}$ lines in the spectrum of Fig. 2(c), both the energy and the intensity of the $809 \mathrm{keV}$ transition seem to be too high to suggest this transition to correspond to the decay of the yet unidentified $1 / 2^{+}$state of ${ }^{137} \mathrm{Sb}$. The large absolute intensity, which corresponds to a $\log f t$ value of 5.2(4) (assuming an excitation energy of $809 \mathrm{keV}$ ), rather suggests this $\gamma$ ray to be emitted in the decay of a strongly populated $0^{-}$state in ${ }^{138} \mathrm{Sb}$, in analogy to the strong $\beta$ feeding of the $0_{1}^{-}$states in ${ }^{134} \mathrm{Sb}$ and ${ }^{136} \mathrm{Sb}$. However, this spin assignment certainly has to be considered very tentative.

\section{DISCUSSION}

In this section, the new experimental results will be compared with, and interpreted with the help of, two different sets of shell-model (SM) calculations. In all calculations, which were performed using the Strasbourg shell-model code ANTOINE [32,33], the valence space includes the full major proton and neutron shells above the closed ${ }^{132} \mathrm{Sn}$ core, i.e., the $0 g_{7 / 2}, 1 d_{5 / 2}, 1 d_{3 / 2}, 2 s_{1 / 2}, 0 h_{11 / 2}$ proton orbitals and the 
$1 f_{7 / 2}, 2 p_{3 / 2}, 2 p_{1 / 2}, 0 h_{9 / 2}, 1 f_{5 / 2}, 0 i_{13 / 2}$ neutron orbitals. The proton and neutron single-particle energies are input parameters which are taken from the experimental spectra of ${ }^{133} \mathrm{Sb}$ [7] and ${ }^{133} \mathrm{Sn}[34,35]$, respectively, except for the still-missing SPE of the $\pi 2 s_{1 / 2}$ and $\nu 0 i_{13 / 2}$ orbitals, which are taken from Refs. [36,37]. As a reference, calculations employing a two-body effective interaction which was derived within the framework of perturbation theory from the CD-Bonn $N N$ potential and renormalized using the $V_{\text {low- } k}$ approach [10] were performed. This well-established interaction, which will be called Napoli in the following, has already demonstrated in the past that it is well suited to describe the properties of $Z \geqslant 50$, $N \geqslant 82$ nuclei. In particular, the experimental information on excited states in ${ }^{134} \mathrm{Sb}$, only one proton and one neutron outside doubly magic ${ }^{132} \mathrm{Sn}$, as well as the surprisingly low position of the $5 / 2^{+}$state in ${ }^{135} \mathrm{Sb}$, are well reproduced by these calculations $[9,38]$. Recently, the same interaction has also been used to predict the evolution of single-particle states beyond ${ }^{132} \mathrm{Sn}$. In this study, a much stronger fragmentation of the single-particle strength is predicted for the $Z=51$ isotopes ${ }^{135} \mathrm{Sb}$ and ${ }^{137} \mathrm{Sb}$ as compared with the $N=83$ isotones ${ }^{135} \mathrm{Te}$ and ${ }^{137} \mathrm{Xe}$, mainly due to the reduced neutron pairing beyond $N=82$ [10]. These reference calculations will be compared with a second set of SM calculations employing the more recent N3LOP interaction, which was derived from the realistic N3LO chiral effective-field theory potentials [39]. The renormalization was again achieved by the so-called low-momentum potential approach $V_{\text {low- } k}$, defined within the cutoff $\lambda=2.2 \mathrm{fm}^{-1}$. In both shell-model calculations, the effective interactions were adapted to the model space by many-body perturbation theory techniques [40], including all $\hat{Q}$-box folded diagrams up to the second order. Multipole and monopole adjustments of the initial version of the N3LOP effective interaction were performed in order to reproduce, respectively, the known isomeric decays in ${ }^{134,136,138} \mathrm{Sn}$, see Refs. [41,42], and the experimental single-particle states of the $N=82,83$ isotones. Here, in the spirit of Zuker [43], the applied two-body monopole corrections mimic the effects of three-body forces, which are missing in the initial realistic interaction. In the past, the N3LOP effective interaction was shown to provide a very good overall description of the spectroscopic properties of a large number of nuclei in the quadrant northeast of ${ }^{132} \mathrm{Sn}$, revealing the presence of large quadrupole collectivity in the $N=86,88$ isotones [44-48].

\section{A. The odd-even Sb isotopes}

Both SM interactions employed in the present work have already been used to investigate the origin of the surprisingly low energy of the $5 / 2^{+}$state in ${ }^{135} \mathrm{Sb}[9,10,46]$. It was shown that this dramatic decrease of the $5 / 2^{+}$energy from $962 \mathrm{keV}$ in ${ }^{133} \mathrm{Sb}$ to $281 \mathrm{keV}$ in ${ }^{135} \mathrm{Sb}$ is mainly due to the reduced neutron pairing above $N=82$ and therefore cannot be interpreted as direct evidence for a strong decrease of the $1 d_{5 / 2}-0 g_{7 / 2}$ proton spacing. Due to the reduced neutron pairing, seniority-3 configurations are low enough in energy in order to significantly contribute to the low-lying states. For example, with the Napoli interaction, a spectroscopic factor

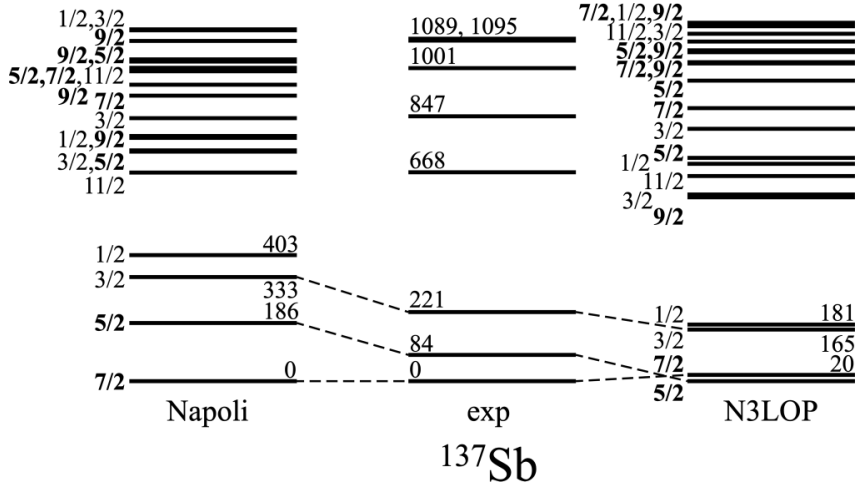

FIG. 6. Comparison between the experimental energies of excited states in ${ }^{137} \mathrm{Sb}$ and SM calculations employing the Napoli (left) and N3LOP (right) interactions, respectively. All calculated states have positive parity.

as low as 0.42 was predicted for the $5 / 2^{+}$state in ${ }^{135} \mathrm{Sb}$ in Ref. [10]. Calculations using the N3LOP interaction were shown to nicely reproduce the experimental energies of the first excited $5 / 2^{+}$states in the $N=82,84 \mathrm{Sb}, \mathrm{I}, \mathrm{Cs}$, and $\mathrm{La}$ isotopes, in particular that in ${ }^{135} \mathrm{Sb}$ [46]. Note that this agreement is not a consequence of the applied monopole correction, it is already obtained with the original realistic interaction. Both calculations predict a further reduction of the $1 d_{5 / 2}-0 g_{7 / 2}$ proton gap when more neutrons are added to the $1 f_{7 / 2}$ shell $[10,46]$. This feature was already discussed in Refs. [47-49]. In the first, the effective SPE were calculated, showing a decrease of the proton gap with increasing neutron number with the inversion taking place at $N=90$. This reduction has been interpreted in Ref. [47] as being due to the coupling between single-particle states and collectivity. A similar behavior was also observed in a larger model space ( $\pi s d g, v p f h$ ) [48] and assessed on the basis of a spin-tensor decomposition [50]. The latter showed that the reduction of the proton gap is mainly caused by the central part, which is counterbalanced by an opposite tensor behavior.

${ }^{137} \mathrm{Sb}$ is considered as one of the key nuclei to pin down the evolution of the single-particle structure beyond $N=82$. In the present work, experimental information on excited states in this nucleus was presented for the first time. The excited-state energies are compared with the results of the two different shell-model calculations employed in this work in Fig. 6. As noted in Sec. III A, the $\beta$ decay of the $N=50$, 51 nuclei is dominated by ff transitions. It is therefore expected that mainly excited states with spins of $5 / 2^{+}, 7 / 2^{+}$, and $9 / 2^{+}$are populated in the decay of the $7 / 2^{-}$ground state of ${ }^{137} \mathrm{Sn}$. While arguments have been put forward for the spin assignments of $\left(7 / 2^{+}\right)$and $\left(5 / 2^{+}\right)$to the ground state and the $84 \mathrm{keV}$ level, respectively, the situation with respect to the state at $221 \mathrm{keV}$ is less clear. In the shell-model calculations, only two additional states, with spins of $3 / 2^{+}$and $1 / 2^{+}$, are expected below an excitation energy of $550 \mathrm{keV}$. States with such spin values should be very weakly populated in the $\beta$ decay of ${ }^{137} \mathrm{Sn}$. Experimentally, the $221 \mathrm{keV}$ state seems to receive significant direct feeding, leading to a $\log f t$ value of 6.3(2). However, considering that unobserved feeding, or 


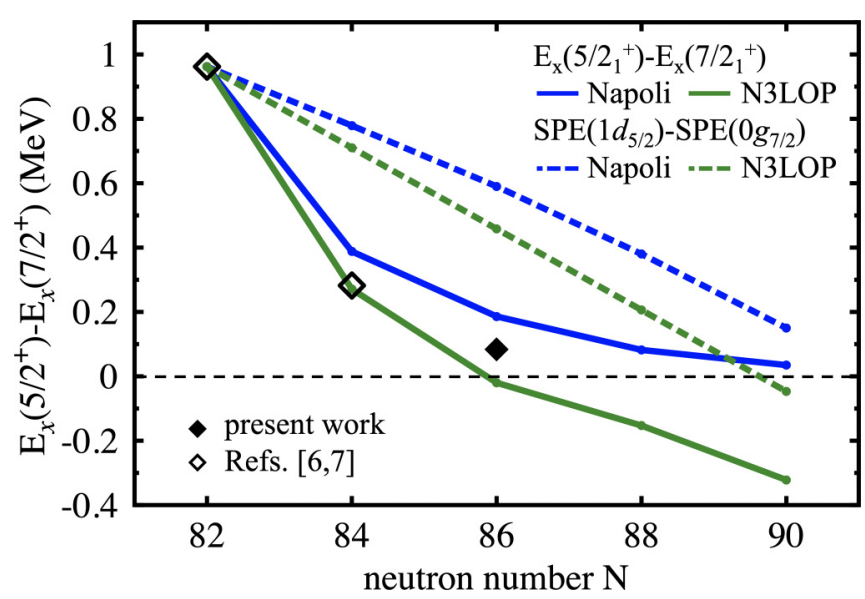

FIG. 7. Excitation energy difference between the $5 / 2_{1}^{+}$and $7 / 2_{1}^{+}$ states in the odd $\mathrm{Sb}$ isotopes. Experimental values are shown as open (from Refs. [7,8]) and filled (present work) diamonds while solid blue (dark gray) [green (light gray)] lines represent the results of the SM calculations employing the Napoli [N3LOP] interactions. The corresponding differences between the effective SPE of the $1 d_{5 / 2}$ and $0 g_{7 / 2}$ orbitals deduced from the SM calculations are shown by dashed lines (taken from Refs. [10,46]).

feeding by $\gamma$ rays which could not be placed in the level scheme, may explain at least part of the apparent direct feeding, we tentatively assign a spin of $\left(3 / 2^{+}\right)$to the $221 \mathrm{keV}$ state. Figure 6 shows that the calculations with the Napoli interaction tend to overestimate the excitation energies of the lowest states in ${ }^{137} \mathrm{Sb}$. The N3LOP calculations, on the other hand, predict a spectrum which is slightly too compressed, including nearly degenerate $5 / 2^{+}$and $7 / 2^{+}$states. The figure also shows that, in both calculations, several states with spins of $5 / 2^{+}, 7 / 2^{+}$, and $9 / 2^{+}$are predicted with excitation energies in the range $0.5-1.2 \mathrm{MeV}$, which could correspond to the experimentally established levels at 668,847, 1001, 1089, and $1095 \mathrm{keV}$.

The excitation energies of the first $5 / 2^{+}$and $3 / 2^{+}$states in the odd $\mathrm{Sb}$ isotopes depend on two factors, the evolution of the effective $0 g_{7 / 2}, 1 d_{5 / 2}$, and $1 d_{3 / 2}$ single-particles energies with increasing neutron number, and the neutron-neutron pairing which determines the excitation energy of seniority3 configurations. In Fig. 7, the experimental and calculated energies of the $5 / 2_{1}^{+}$states in the odd $\mathrm{Sb}$ isotopes, relative to those of the $7 / 2_{1}^{+}$levels, are shown. Also included in this figure are the differences between the $1 d_{5 / 2}$ and $0 g_{7 / 2}$ proton SPE which were deduced from the SM calculations following the prescription given in Ref. [10]. Clearly, between ${ }^{133} \mathrm{Sb}$ and ${ }^{135} \mathrm{Sb}$ the $5 / 2_{1}^{+}$energy drops much faster than the SPE difference in both calculations. This is due to the admixture of seniority- 3 configurations to the wave function of the $5 / 2_{1}^{+}$ state in ${ }^{135} \mathrm{Sb}$. We note that the occupancy of the proton $1 d_{5 / 2}$ single-particle orbital is only around $60 \%$. Then, between ${ }^{135} \mathrm{Sb}$ and ${ }^{137} \mathrm{Sb}$, the decrease of the $5 / 2_{1}^{+}$energy softens considerably. In the SM calculations, the $1 d_{5 / 2}$ occupancy increases to values around $79 \%$ and one may conclude that the decrease of the $1 d_{5 / 2}-0 g_{7 / 2}$ SPE difference becomes more relevant for the further drop of the $5 / 2_{1}^{+}$energy. In general, the decrease of the $5 / 2_{1}^{+}$energy is more pronounced in the N3LOP calculations, probably in part due to the reduction of the $1 f_{7 / 2}$ neutron-neutron pairing matrix element, which was applied in the modification of the original N3LO interaction. The experimental energy of the $\left(5 / 2_{1}^{+}\right)$state in ${ }^{137} \mathrm{Sb}$, $E_{x}=84 \mathrm{keV}$, lies in the middle between the two calculated values and confirms the change of slope predicted by the calculations. Finally, we note that, while the effective singleparticle energy of the $1 d_{5 / 2}$ orbital obtained by employing the N3LOP interaction becomes lowest for the first time in ${ }^{141} \mathrm{Sb}$, the same interaction predicts that the $5 / 2_{1}^{+}$level possibly forms the ground state already in ${ }^{139} \mathrm{Sb}$, which seems to be confirmed by extrapolating the experimental trend (see Fig. 7). This discordance could be interpreted, as discussed above, as an effect of the neutron-neutron interaction. The same effect, although less evident, is shown by the results of the Napoli calculations. In fact, even if in this case the differences between the $5 / 2_{1}^{+}$and $7 / 2_{1}^{+}$states and the effective single-particle energies of the $1 d_{5 / 2}$ and $0 g_{7 / 2}$ orbitals are both larger than zero up to ${ }^{141} \mathrm{Sb}$, we see that the curve corresponding to the excitation-energy differences lies always below that of the effective single-particle energies.

\section{B. The odd-odd Sb isotopes}

In ${ }^{134} \mathrm{Sb}$, with one proton and one neutron outside doubly magic ${ }^{132} \mathrm{Sn}$, all members of the $\pi 0 g_{7 / 2} \otimes \nu 1 f_{7 / 2}$ multiplet, which is lowest in energy, and also the second $1^{-}$and $2^{-}$ states, which are members of the $\pi 1 d_{5 / 2} \otimes v 1 f_{7 / 2}$ multiplet, are experimentally known [8]. In Figs. 8(a) and 8(d), the experimental energies are compared with the results of the two SM calculations performed in the present work. As already discussed in Ref. [38], the Napoli interaction very nicely reproduces the experimental excitation energies, but this is also true for the N3LOP interaction. In both calculations, the lowest states of all spins between $0^{-}$and $7^{-}$have a contribution of at least $83 \%$ of the $\pi 0 g_{7 / 2} \otimes \nu 1 f_{7 / 2}$ configuration to the wave function and $\pi 0 g_{7 / 2}$ occupancies of $89 \%-100 \%$. The wave functions of the second $1^{-}-6^{-}$states are in most cases dominated by the $\pi 1 d_{5 / 2} \otimes \nu 1 f_{7 / 2}$ configuration. For the $3_{2}^{-}$ and $5_{2}^{-}$states, however, the $\pi 0 g_{7 / 2} \otimes \nu 2 p_{3 / 2}$ configuration has the strongest contribution $\left[45 \%(48 \%)\right.$ for the $3_{2}^{-}$and $76 \%(81 \%)$ for the $5_{2}^{-}$levels with the Napoli (N3LOP) interaction]. In addition to the excitation energies, the occupancies of the odd proton in the $0 g_{7 / 2}$ orbital for the first state of each spin and the $1 d_{5 / 2}$ orbital for the second level of each spin are also included in Fig. 8. The above-mentioned contribution of the $\pi 0 g_{7 / 2} \otimes \nu 2 p_{3 / 2}$ configuration to the $3_{2}^{-}$and $5_{2}^{-}$states is reflected by low proton occupations of the $1 d_{5 / 2}$ orbital for these states [see Figs. 8(a) and 8(d)].

In the next odd-odd isotope ${ }^{136} \mathrm{Sb}$, states with spins of $1^{-}$, $2^{-}, 4^{-}$, and $6^{-}$were proposed in Refs. [22-24]. In the present work, spin values of $0^{-}$and $1^{-}$were tentatively assigned to the newly established states with excitation energies of 535 and $674 \mathrm{keV}$, respectively. The comparison of this experimental information to the two different SM calculations is shown in Figs. 8(b) and 8(e). The first observation is that the two extra neutrons in ${ }^{136} \mathrm{Sb}$, as compared with ${ }^{134} \mathrm{Sb}$, lead to a significant compression of the excitation spectrum, 


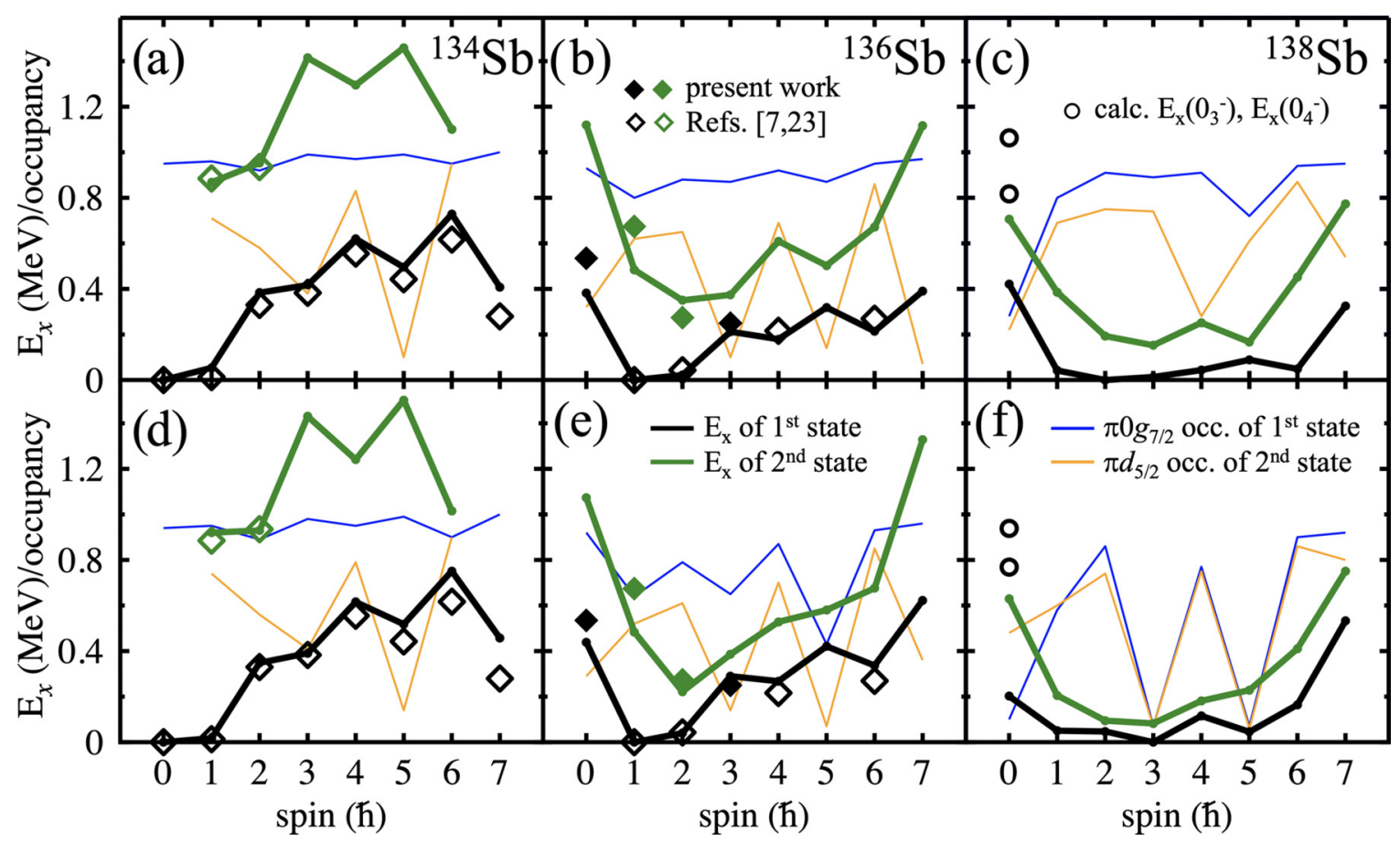

FIG. 8. Calculated first (black) and second [green (dark gray)] states in the spin range $0^{-}-7^{-}$in the odd-odd isotopes ${ }^{134,136,138} \mathrm{Sb}$. Results of SM calculations using the Napoli interaction are shown in parts (a), (b), and (c) while those obtained with the N3LOP interaction are shown in parts (d), (e), and (f). Experimentally proposed states in ${ }^{134,136} \mathrm{Sb}$ are shown as open (from Refs. [8,24]) and filled (present work) diamonds. Thin lines indicate the proton occupancy of the $0 g_{7 / 2}$ orbital for the first states of each spin [blue (dark gray)] and the $1 d_{5 / 2}$ orbital for the second state of each spin [orange (light gray)]. For ${ }^{138} \mathrm{Sb}$, the excitation energies of the third and fourth calculated $0^{-}$states are included as circles.

both within the group of first states of each spin as well as between the first and the second states of each spin. Note that this trend is in accord with that observed for the $5 / 2_{1}^{+}-7 / 2_{1}^{+}$ spacing in the odd isotopes in Fig. 7. In ${ }^{136} \mathrm{Sb}$, the first state of each spin is dominated by the $\pi 0 g_{7 / 2} \otimes \nu\left(1 f_{7 / 2}\right)^{3}$ configuration, which is contributing with $57 \%-75 \%(30 \%-70 \%)$ in the Napoli (N3LOP) calculations, with the only exception of the $5_{1}^{-}$state, for which the N3LOP calculations predict a larger contribution by the $\pi 1 d_{5 / 2} \otimes v\left(1 f_{7 / 2}\right)^{3}$ configuration (34\% as compared with $30 \%$ ). This decrease in the weight of the main configuration translates into a slight decrease of the $\pi 0 g_{7 / 2}$ occupancy. For the second-excited states of each spin, significant mixing is observed, which results in $\pi 1 d_{5 / 2}$ occupancies of only $62 \%-86 \%(52 \%-85 \%)$ for the $11_{2}^{-}, 2_{2}^{-}$, $4_{2}^{-}$, and $6_{2}^{-}$states. For the $3_{2}^{-}$and $5_{2}^{-}$levels, this value further drops to $10 \%-14 \%(7 \%-14 \%)$ due to a strong contribution of the $\pi 0 g_{7 / 2} \otimes v 2 p_{3 / 2}\left(1 f_{7 / 2}\right)^{2}$ partition. Figures $8(\mathrm{~b})$ and $8(\mathrm{e})$ show that both calculations rather nicely reproduce the position and the shape of the lowest multiplet. In particular, they reproduce the experimentally observed odd-even staggering, which interestingly is opposite to the one observed in ${ }^{134} \mathrm{Sb}$ [see Figs. 8(a) and $8(\mathrm{~d})$ ], and the increase of the excitation energy of the first $0^{-}$level as compared with that in ${ }^{134} \mathrm{Sb}$. The calculations suggest tentative spin assignments of $\left(3_{1}^{-}\right)$and $\left(2_{2}^{-}\right)$for the newly established levels at 250 and $274 \mathrm{keV}$, respectively. Such assignments are consistent with the observed decays to the $\left(1_{1}^{-}\right)$and $\left(2_{1}^{-}\right)$states in the first case and the $\left(1_{1}^{-}\right)$level in the second. A spin value of $\left(3_{1}^{-}\right)$ for the $250 \mathrm{keV}$ state is also consistent with the experimental finding that this level is not populated in the decay of the state at $2641 \mathrm{keV}$. This highly excited state is populated with a low $\log f t$ value of 5.2(1) indicative of an allowed decay. As discussed in Sec. III A, the only relevant GT decay in this region is the $\nu 0 h_{9 / 2} \rightarrow \pi 0 h_{11 / 2}$ transition, which in the case of the ${ }^{136} \mathrm{Sn}$ decay is only enabled by small admixtures (around $8 \%$ ) to the ground-state wave function in which one neutron pair is excited from the $1 f_{7 / 2}$ to the $0 h_{9 / 2}$ orbital. Since only $1^{+}$states in ${ }^{136} \mathrm{Sb}$ can be populated by this spinflip GT decay, we tentatively assign this spin to the new $2641 \mathrm{keV}$ level. In the SM calculations with the Napoli (N3LOP) interaction, the first $1^{+}$level is dominated by configurations with the proton in the $0 g_{7 / 2}$ orbital. However, the third (fourth) $1^{+}$state at an excitation energy of $3.28 \mathrm{MeV}(3.5$ $\mathrm{MeV}$ ) has a strong (48\% and $35 \%$, respectively) contribution of the $\pi 0 h_{11 / 2} \otimes \nu 0 h_{9 / 2}\left(1 f_{7 / 2}\right)^{2}$ configuration and thus could be the theoretical counterpart of the $2641 \mathrm{keV}$ state.

The good overall agreement between experiment and calculations for ${ }^{134} \mathrm{Sb}$ and ${ }^{136} \mathrm{Sb}$ motivated us to extend the calculations to the next odd-odd isotope, ${ }^{138} \mathrm{Sb}$, for which unfortunately only very little experimental information could be obtained in the present work. As shown in Figs. 8(c) and $8(\mathrm{f})$, the compression of the calculated spectra, already observed when going from ${ }^{134} \mathrm{Sb}$ to ${ }^{136} \mathrm{Sb}$, is even more pronounced when two more neutrons are added. For ${ }^{138} \mathrm{Sb}$, both calculations predict as many as six states with excitation energies below $100 \mathrm{keV}$. For this nucleus, significant 
differences are found between the two SM calculations. In the calculations with the Napoli interaction, the first states with spin 1-7 are still dominated by configurations with the odd proton occupying the $0 g_{7 / 2}$ orbital (occupancies of $72 \%-95 \%$, contribution of the $\pi 0 g_{7 / 2} \otimes v 1\left(f_{7 / 2}\right)^{5}$ partition in the range $37 \%-54 \%$ ). In the second-excited state of each spin, the proton mainly occupies the $1 d_{5 / 2}$ orbital, with the only exception being the $4_{2}^{-}$level. With the N3LOP interaction, the picture is clearly different. In these calculations, the first $3^{-}$and $5^{-}$states are based on the proton in the $1 d_{5 / 2}$ orbital while in all other first levels in the spin range $1-7$ the proton occupies mainly the $0 g_{7 / 2}$ orbital. It is interesting to note that, although in this calculation the $0 g_{7 / 2}$ and $1 d_{5 / 2}$ proton configurations are nearly degenerate and the first and second states are therefore very close in energy, the proton occupancy clearly distinguishes the two sets of states [see occupancies in Fig. 8(f)]. In the N3LOP calculation, the $\pi 1 d_{5 / 2}$-dominated $3^{-}$state forms the ground state in ${ }^{138} \mathrm{Sb}$ while the calculations with the Napoli interaction predict a $\pi 0 g_{7 / 2}$-dominated $2^{-}$ground state for this $N=87$ isotope. These predictions for ${ }^{138} \mathrm{Sb}$ are consistent with those of the $5 / 2^{+}\left(7 / 2^{+}\right)$ground-state spins for the odd neighbors ${ }^{137,139} \mathrm{Sb}$ obtained with the N3LOP (Napoli) interactions; see Fig. 7. Considering the very small energy differences between the calculated first-excited states, it is unfortunately at present not possible to make a reliable prediction for the ground-state spin of ${ }^{138} \mathrm{Sb}$. As will be discussed in detail in Ref. [30], the available experimental information for the $\beta$ decay of this nucleus, which was obtained in the same experiment, suggests a ground-state spin in the range $\left(3^{-}, 4^{-}, 5^{-}\right)$.

As reported in Sec. III C, a $\gamma$ ray with an energy of $809 \mathrm{keV}$ was observed in the $\beta$ decay of ${ }^{138} \mathrm{Sn}$. Based on the low $\log f t$ value deduced from its absolute intensity, it was suggested that it depopulates a $0^{-}$state in ${ }^{138} \mathrm{Sb}$, in analogy to the strong population of the $0^{-}$states in ${ }^{134,136} \mathrm{Sb}$ in the $\beta$ decays of ${ }^{134,136} \mathrm{Sn}$. In these decays, $0^{-}$states are populated via the $v 1 f_{7 / 2} \rightarrow \pi 0 g_{7 / 2}$ spin-flip transition. Therefore, the odd proton necessarily has to occupy the $0 g_{7 / 2}$ orbital in these $0^{-}$levels. In the two SM calculations, the first $0^{-}$ states in ${ }^{134,136} \mathrm{Sb}$ indeed show $0 g_{7 / 2}$ occupancies above $92 \%$ and contributions of the $\pi 0 g_{7 / 2} \otimes \nu 1 f_{7 / 2}^{n}$ configuration of at least $56 \%$. For ${ }^{138} \mathrm{Sb}$, however, the calculations predict sizable contributions of $0 g_{7 / 2}$ protons only for the second (Napoli) and third (N3LOP) $0^{-}$states at excitation energies of 707 and $768 \mathrm{keV}$, respectively. In contrast, in the first calculated $0^{-}$ levels at 421 and $202 \mathrm{keV}$, respectively, the proton is predominantly occupying the $1 d_{5 / 2}$ orbital. The relatively high energy of the most intense $\gamma$ ray emitted following the $\beta$ decay of ${ }^{138} \mathrm{Sn}, E_{\gamma}=809 \mathrm{keV}$, therefore seems to indirectly confirm the scenario predicted by the calculations.

\section{CONCLUSIONS}

We reported on a first study of the $\beta$ decays of ${ }^{136,137,138} \mathrm{Sn}$ which was performed at the RIBF facility at the RIKEN Nishina Center within the EURICA campaign. The clean ion identification and the high $\gamma$-ray detection efficiency of the present experiment allowed us to considerably extend the excitation scheme of ${ }^{136} \mathrm{Sb}$. The new experimental information confirms the energy of $43 \mathrm{keV}$ for the first excited state in this nucleus and thus resolves the conflict between the results of two previous experiments using isomer spectroscopy. For the $N=86$ isotope ${ }^{137} \mathrm{Sb}$, the decay of excited states was observed for the first time and, based on $\gamma \gamma$ coincidence information and energy sums, a level scheme comprising eight excited states was proposed. The experimental results were compared with systematic shell-model calculations using two different realistic effective interactions. The observation of the low-lying states in ${ }^{137} \mathrm{Sb}$, together with the available experimental information for the odd-odd isotopes ${ }^{136,138} \mathrm{Sb}$, allowed us to trace the evolution of the $0 g_{7 / 2}$ and $1 d_{5 / 2}$ proton single-particle energies when more and more neutrons are added above the $N=82$ shell closure.

\section{ACKNOWLEDGMENTS}

We thank the staff of the RIKEN Nishina Center accelerator complex for providing high-intensity beams to the experiment. This work was supported by the Spanish Ministerio de Ciencia e Innovación under contracts FPA2014-57196-C5-4-P and FPA2017-84756-C4-2-P and the UK Science and Technology Facilities Council under Grant No. ST/P005101/1. Zs.V. was supported by the European Regional Development Fund (Contract No. GINOP-2.3.315-2016-00034), the National Research Development and Innovation Fund of Hungary (Grant No. K128947), and by RIKEN as short-term International Program Associate.
[1] J. P. Schiffer et al., Phys. Rev. Lett. 92, 162501 (2004).

[2] M.-G. Porquet, S. Peru, and M. Girod, Eur. Phys. J. A 25, 319 (2005).

[3] H. Grawe, K. Langanke, and G. Martínez-Pinedo, Rep. Prog. Phys. 70, 1525 (2007).

[4] O. Sorlin and M.-G. Porquet, Prog. Part. Nucl. Phys. 61, 602 (2008).

[5] Y. Utsuno et al., JPS Conf. Proc. 6, 010007 (2015).

[6] https://www.nndc.bnl.gov/.

[7] M. Sanchez-Vega, B. Fogelberg, H. Mach, R. B. E. Taylor, A. Lindroth, J. Blomqvist, A. Covello, and A. Gargano, Phys. Rev. C 60, 024303 (1999).
[8] J. Shergur et al., Phys. Rev. C 71, 064321 (2005).

[9] L. Coraggio, A. Covello, A. Gargano, and N. Itaco, Phys. Rev. C 72, 057302 (2005).

[10] L. Coraggio, A. Covello, A. Gargano, and N. Itaco, Phys. Rev. C 87, 034309 (2013).

[11] S. Nishimura et al., Prog. Theor. Exp. Phys. 2012, 03 C006 (2012); RIKEN Accel. Prog. Rep. 46, 182 (2013).

[12] P.-A. Söderström et al., Nucl. Instrum. Methods Phys. Res., Sect. B 317, 649 (2013).

[13] T. Kubo et al., Prog. Theor. Exp. Phys. 2012, 03 C003 (2012).

[14] N. Fukuda et al., Nucl. Instrum. Methods Phys. Res., Sect. B 317, 323 (2013). 
[15] J. Eberth et al., Nucl. Instrum. Methods Phys. Res., Sect. A 369, 135 (1996).

[16] J. Simpson, Z. Phys. A: Hadrons Nucl. 358, 139 (1997).

[17] J. Taprogge et al., Phys. Rev. Lett. 112, 132501 (2014).

[18] J. Taprogge et al., Phys. Rev. C 91, 054324 (2015).

[19] A. Jungclaus et al., Phys. Rev. C 93, 041301(R) (2016).

[20] A. Jungclaus et al., Phys. Rev. C 94, 024303 (2016).

[21] G. Lorusso et al., Phys. Rev. Lett. 114, 192501 (2015).

[22] M. N. Mineva et al., Eur. Phys. J. A 11, 9 (2001).

[23] G. S. Simpson, J. C. Angelique, J. Genevey, J. A. Pinston, A. Covello, A. Gargano, U. Koster, R. Orlandi, and A. Scherillo, Phys. Rev. C 76, 041303(R) (2007).

[24] R. Lozeva et al., Phys. Rev. C 92, 024304 (2015).

[25] H. Mach et al., Acta Phys. Pol. B 38, 1213 (2007).

[26] M. Wang et al., Chin. Phys. C 41, 030003 (2017).

[27] J. Hardy et al., Phys. Lett. B 71, 307 (1977).

[28] J. Shergur et al., Phys. Rev. C 65, 034313 (2002).

[29] O. Arndt et al., Phys. Rev. C 84, 061307(R) (2011).

[30] B. Moon et al. (unpublished).

[31] P. Hoff, B. Ekström, and B. Fogelberg, Z. Phys. A 332, 407 (1989).

[32] E. Caurier and F. Nowacki, Acta Phys. Pol. B 30, 705 (1999).

[33] E. Caurier, G. Martínez-Pinedo, F. Nowacki, A. Poves, and A. P. Zuker, Rev. Mod. Phys. 77, 427 (2005).
[34] P. Hoff et al., Phys. Rev. Lett. 77, 1020 (1996).

[35] K. L. Jones et al., Nature (London) 465, 454 (2010).

[36] W. T. Chou and E. K. Warburton, Phys. Rev. C 45, 1720 (1992).

[37] W. Urban et al., Eur. Phys. J. A 5, 239 (1999).

[38] L. Coraggio, A. Covello, A. Gargano, and N. Itaco, Phys. Rev. C 73, 031302(R) (2006).

[39] D. R. Entem and R. Machleidt, Phys. Rev. C 68, 041001(R) (2003).

[40] M. Hjorth-Jensen, T. T. S. Kuo, and E. Osnes, Phys. Rep. 261, 125 (1995).

[41] G. S. Simpson et al., Phys. Rev. Lett. 113, 132502 (2014).

[42] H. Naïdja, F. Nowacki, and K. Sieja, Acta Phys. Pol., B 46, 669 (2015).

[43] A. P. Zuker, Phys. Rev. Lett. 90, 042502 (2003).

[44] H. Naïdja et al., Phys. Rev. C 95, 064303 (2017).

[45] H. Naïdja, F. Nowacki, and B. Bounthong, Phys. Rev. C 96, 034312 (2017).

[46] H. Naïdja and F. Nowacki, J. Phys.: Conf. Ser. 966, 012061 (2018).

[47] H. Naïdja and F. Nowacki, EPJ Web Conf. 193, 01005 (2018).

[48] R. Lozeva et al., Phys. Rev. C 98, 024323 (2018).

[49] R. Lozeva et al., Phys. Rev. C 93, 014316 (2016).

[50] N. Smirnova et al., Phys. Lett. B 686, 109 (2010). 